\title{
Smoothing finite-element investigation of sheet forming limit
}

\author{
Zhe Wang ${ }^{1, a^{*}}$, Cailing $\mathrm{Li}^{1, \mathrm{~b}}$, Xiaoyu Shen ${ }^{1, \mathrm{c}}$,Chao Cui ${ }^{1}$, Jianchao Han ${ }^{1}$, \\ Shaohua Wang ${ }^{1}$,Xiaoming Lai ${ }^{1}$,Jiaowen Liu ${ }^{1}$ \\ ${ }^{1}$ Beijing spacecrafts, Beijing,China ${ }^{1}$ \\ awzh425@126.com, ${ }^{\mathrm{b}}$ lindali6@126.com, ${ }^{\mathrm{c} x i a o y u . s h e n @ 163 . c o m ~}$
}

\begin{abstract}
Keywords: Sheet metal forming, smoothing finite-element method, ductile fracture criterion Abstract. The ductile fracture criterion (DFC) is widely used to predict the forming limit of sheet metal, and the material constants in the criterion need to be determined by the destructive tests. In order to increase the DFC predication accuracy, the key factor is improving the quality of material constants in the DFC. The smoothing finite-element method is developed to obtain the real fracture strain and the accurate material constants. Calculations are carried out for various strain paths of elliptical hydro-bulging process. The result demonstrates SFER method improved the accuracy of the DFC's prediction.
\end{abstract}

\section{Introduction}

The material constants of the ductile fracture criterion are always determined by the following methods. The first is the test method. Major fracture strains are obtained from thickness measurements of fractured grids in combination with minor strains from grids adjacent to fracture, and using the assumption of plastic incompressibility $[1,2,3]$. The second approach is numerical method. The finite element simulation is conducted until the least thickness of model equals to the thickness measured in the fracture position of the specimens. Then the material constants can be calculated considering the simulated strain and stress fields at this moment as the fracture strain and stress fields in the experiment [4]. Both methods need to measure the thickness in the fracture site. However, it's difficult to accurately determine the fracture thickness for the distortions and collapse of the sheet.

To overcome these drawbacks in the previous methods, the smoothing finite-element method [5] is used in this study to quantify the material constants in the DFC. Using the finite element concepts, the limit strains on the fracture edge can be determined by experimental data away from the crack where the data are more reliable. The material constants are determined by smoothing finite-element method, and the DFC is employed and combined with LS-DYNA simulation of the elliptical hydro-bulging process to predict the bulge height of sheet metals, and the validity of the approach is examined by comparing with the measured bulge height. The result demonstrates smoothing finite-element method's effectiveness for improving the accuracy of the prediction of DFC.

\section{Smoothing finite-element method for determining the limit strain}

Smoothing finite-element representation (SFER) [5] method developed here is very suited for filtering noisy measured data, using measured data from arbitrary locations to accommodate the limit strain along the crack edge. Consider a $2 \mathrm{D}$ or $3 \mathrm{D}$ field throughout some region $R$ adjacent to the crack. The smoothing functions of the principle strain $\varepsilon_{1}(\mathrm{x}, \mathrm{y})$ and $\varepsilon_{2}(\mathrm{x}, \mathrm{y})$ can be obtained by 
minimizing the following vector functional:

$$
\{\phi\}=\left\{\begin{array}{l}
\phi_{1}\left(\varepsilon_{1}\right) \\
\phi_{2}\left(\varepsilon_{2}\right)
\end{array}\right\}=\left\{\begin{array}{c}
\frac{1}{2} \sum_{i=1}^{T} \alpha_{i}\left[\varepsilon_{1}\left(x_{i}, y_{i}\right)-\hat{\varepsilon}_{1 \mathrm{i}}\right]^{2}+\frac{\lambda}{2} \int_{R}\left[\varepsilon_{1, x x}{ }^{2}+2 \varepsilon_{1, x y}{ }^{2}+\varepsilon_{1, y y}{ }^{2}\right] d R \\
\frac{1}{2} \sum_{i=1}^{T} \alpha_{i}\left[\varepsilon_{2}\left(x_{i}, y_{i}\right)-\hat{\varepsilon}_{2 \mathrm{i}}\right]^{2}+\frac{\lambda}{2} \int_{R}\left[\varepsilon_{2, x x}{ }^{2}+2 \varepsilon_{2, x y}{ }^{2}+\varepsilon_{2, y y}{ }^{2}\right] d R
\end{array}\right\}
$$

where $\phi_{1} 、 \phi_{2}$ are quadratic positive definite functions, $\varepsilon_{1}$ and $\varepsilon_{2}$ are predicted piecewise twice-differentiable functions defined in the region, $R, \hat{\varepsilon}_{1 \mathrm{i}}$ and $\hat{\varepsilon}_{2 \mathrm{i}}$ are measured values of the input strains at location, $\left(x_{i}, y_{i}\right), T$ is the total number of locations in region $R$ at which one inputs one or two strain values, $\alpha_{i}$ is the weighting factor of the data point $i, \lambda$ is a smoothing parameter. One usually uses $\alpha_{i}=1$.

An appropriate approximate solution of the above can be developed using finite element concepts. The region $R$ is discretized by the eight-node isoparametric quadrilateral elements. The global coordinates and strain within such elements are defined as follows:

$$
\begin{aligned}
& \left\{\begin{array}{l}
x \\
y
\end{array}\right\}=[N]\{c\} . \\
& \left\{\begin{array}{l}
\varepsilon_{1} \\
\varepsilon_{2}
\end{array}\right\}=[N]\{\varepsilon\} .
\end{aligned}
$$

Substituting Eqs. 2 and 3 into Eq. 1and minimizing gives:

$$
\left\{\frac{\partial \phi_{1}\left(\varepsilon_{1}\right)}{\partial\{D\}}, \frac{\partial \phi_{2}\left(\varepsilon_{2}\right)}{\partial\{D\}}\right\}^{T}=\{0\} .
$$

Where $\{D\}$ contains the strain at all nodes throughout the region $R$. Eq. 4 leads to

$$
\begin{aligned}
& \sum_{j=1}^{M}\left[\sum_{i=1}^{J} \alpha_{i}\left[N\left(\xi_{i}, \eta_{i}\right)\right]_{j}^{T}\left[N\left(\xi_{i}, \eta_{i}\right)\right]_{j}\{d\}_{j}+\lambda \int_{R_{j}}\left\{\left[N_{, x x}\right]_{j}^{T}\left[N_{, x x}\right]_{j}+2\left[N_{, x y}\right]_{j}^{T}\left[N_{, x y}\right]_{j}\right.\right. \\
& \left.\left.\left.+\left[N_{, y y}\right]_{j}^{T}\left[N_{, y y}\right]_{j}\right\} d R\{d\}\right\}_{j}\right]=\sum_{j=1}^{M} \sum_{i=1}^{J} \alpha_{i}\left[N\left(\xi_{i}, \eta_{i}\right)\right]_{j}^{T}\left\{\hat{\varepsilon}_{1 \mathrm{i}}, \hat{\varepsilon}_{2 \mathrm{i}}\right\}
\end{aligned}
$$

Where $\left(\xi_{i}, \eta_{i}\right)$ are the isoparametric (local) coordinates of the locations of an measured input value $i, J$ is the total number of locations in element $j, R_{j}$ is the region of the $j$ th element, $M$ is the total number of elements in region $R,\{d\}_{j}$ is the nodal strain vector in global coordinates of the $j$ th element, and $\left[N\left(\xi_{i}, \eta_{i}\right)\right]$ is the shape-function matrix evaluated at locations $\left(\xi_{i}, \eta_{i}\right)$. Solving Eq. 5 for $\{D\}$ determines all the nodal strain including the limit strain along the edge of the crack.

\section{Experimental and analytical procedures}

Experimental procedure. The material used in this study is mild steel sheet $08 \mathrm{Al}(\mathrm{ZF})$, at a nominal gauge of $0.8 \mathrm{~mm}$. The various uniaxial material parameters are given in Table 1. In addition to the uniaxial tension tests, elliptical hydro-bulging tests with four aspect ratios are carried out. The major axis (A) of the elliptical die with the diameter of $102 \mathrm{~mm}$ is fixed, and the minor axis (B)/major axis (A) are 1.0, 0.9, 0.8, and 0.6 respectively. 
Table 1 Tensile properties of material

\begin{tabular}{c|c|c|c|c|}
\hline Material & $\begin{array}{c}K \text {-value[Mpa] } \\
\left(\sigma=K \varepsilon^{n}\right)\end{array}$ & $\begin{array}{c}\text { Work-hardening } \\
\text { exponent, } n\end{array}$ & $\begin{array}{l}\text { Normal anisotropy } \\
\text { parameter, } r\end{array}$ & $\begin{array}{c}\text { Tensile strength } \\
\text { [Mpa] }\end{array}$ \\
\hline $08 \mathrm{Al}(\mathrm{ZF})$ & 553.47 & 0.252 & 1.5 & 270 \\
\hline
\end{tabular}

Smoothing finite-element determination of the material constants of ductile fracture criterion.

In this study, the following criterion proposed by Oyane et al. [6] is employed to predict the forming limit of sheet metal hydro-bulging process:

$$
\int_{0}^{\varepsilon_{\mathrm{f}}}\left(\frac{\sigma_{\mathrm{m}}}{\bar{\sigma}}+\mathrm{a}\right) \mathrm{d} \bar{\varepsilon}=\mathrm{b} \text {. }
$$

Where $\bar{\varepsilon}_{f}$ is the effective strain at fracture site, $a$ and $b$ are material constants. In present study, the material constants are determined by uniaxial tension and circle hydro-bulging tests. In order to obtain the real limit strain, the SFER method is used to represent the strain field of the destructive tests, see Fig1 and 2. Table 2 compares the fracture strains obtain by the SFER method and by the test method.
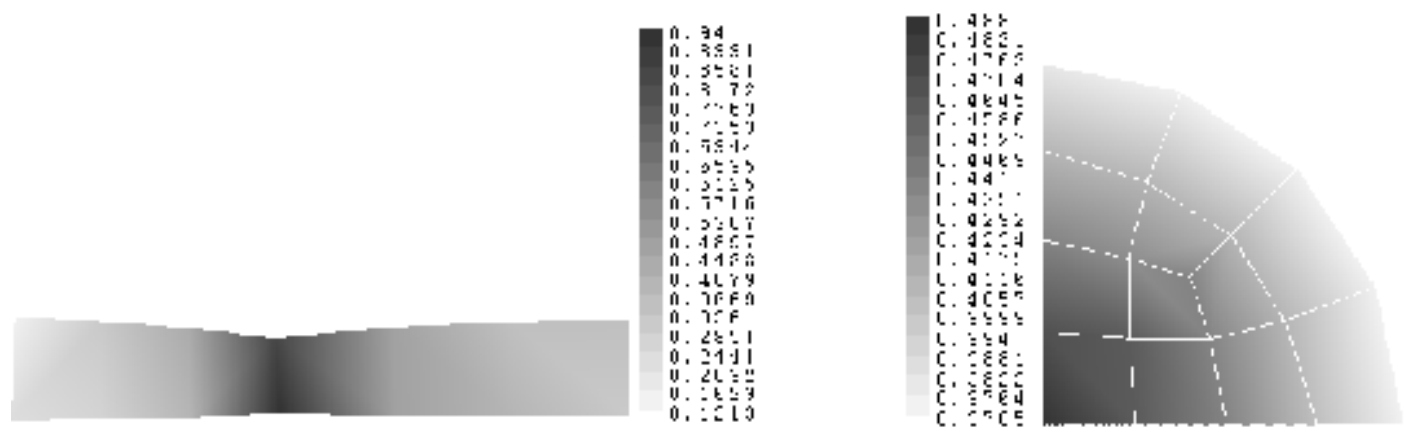

Fig. $11^{\text {st }}$ principle strain field of uniaxial tension and circle hydro-bulging tests obtained by SFER method.

According to Hill's quadratic normal anisotropic plastic potential [7], hydrostatic stress component and effective strain can be expressed as the functions of the ratio of the plastic strain increments $\rho\left(=\mathrm{d} \varepsilon_{2} / \mathrm{d} \varepsilon_{1}\right)$.

$$
\begin{aligned}
& \frac{\sigma_{m}}{\bar{\sigma}}=\frac{\sqrt{1+2 r}(1+\rho)}{3 \sqrt{1+\left(\frac{2 r}{1+r}\right) \rho+\rho^{2}}} . \\
& d \bar{\varepsilon}=\frac{1+r}{\sqrt{1+2 r}} \sqrt{1+\left(\frac{2 r}{1+r}\right) \rho+\rho^{2}} d \varepsilon_{1} .
\end{aligned}
$$

Provided that the strain ratios, $\rho$ are constant during deformation until the fracture initiation, the material constants $a$ and $b$ can be obtained by Eqs.6-8 and fracture strain in the two different stress conditions. Table 2 compares the fracture strains and material constants obtain by the SFER method and by the test method.

Table $21^{\text {st }}$ principle fracture strain $\varepsilon_{1 f}$ obtained from SFER method and the test method

\begin{tabular}{c|c|c|c|c|}
\hline & $\varepsilon_{1 f}$ (uniaxial) & $\begin{array}{c}\varepsilon_{1 f} \text { (circle } \\
\text { hydro-bulging) }\end{array}$ & $a$ & $b$ \\
\hline The test method & 0.76 & 0.43 & -2.3 & -1.5 \\
\hline SFER method & 0.94 & 0.488 & -3.3 & -2.8 \\
\hline
\end{tabular}




\section{Summary}

Fig. 3 shows the comparison between the predicted and experimental critical bulge height for different aspect rations. Marks 1-4 in the horizontal axis indicates the aspect ratio $\mathrm{B} / \mathrm{A}=1.0,0.9,0.8$, 0.6 respectively. It is found that the ductile fracture criterion can predict the forming limit of hydro-bulging process successfully, and the prediction obtained by SFER method is more approaching to the experimental result compared with the test method.

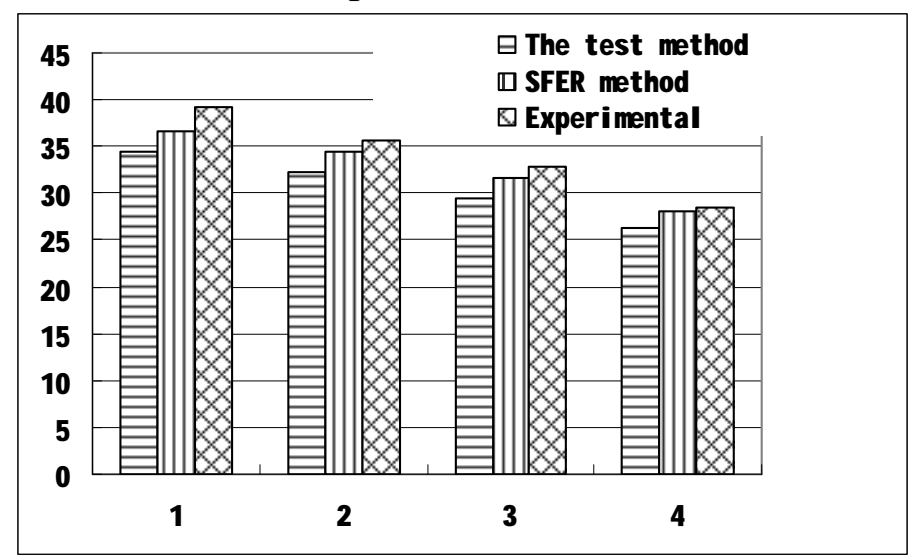

Fig. 3 Comparison between the calculated and experimental critical bulging height

It may be expected that the present approach enable the determination of the sheet metal's material constants for the ductile fracture accurately, which is meaningful to operate valuable estimation of the ductile fracture criterion.

\section{References}

[1] M. Jain, J. Allin, D.J. Lloyd: Int J Mech Sci. Vol.41 (1999), p.1273-1288

[2] H. Takuda, K. Mori, N. Takakura, K. Yamaguchi: Int J Mech Sci. Vol.42 (2000), p. 785-798

[3] H. Takuda, K. Mori, H. Fujimoto, N. Hatta: J Mater Process Tech. Vol.92-93 (1999), p.433-438

[4] Young-Woong Lee, Jeffrey C. Woertz, Tomasz Wierzbicki: Int J Mech Sci. Vol.46 (2004), p.751-781

[5] Z H Feng, R E Rowlands: Comput Struct. Vol.26 (1987), p. 979-990

[6] M. Oyane, T. Sato, K. Hosono, A. Takezoe: J Mech Working Tech. Vol.4 (1980), p.65-81

[7] R Hill: The Mathematical Theory of Plasticity (Oxford University Press, Oxford, 1950) 